\title{
Distribution of homobaric and heterobaric leafed species in the Brazilian Cerrado and seasonal semideciduous forests
}

\section{Katiane Reis Mendes, Silvia Rodrigues Machado, Amanda Cristina Esteves Amaro, Stefany Cristina Melo Silva, Valdemir Ferreira Júnior, Tatiane Maria Rodrigues*}

Univ. Estadual Paulista, UNESP, Institute of Biosciences of Botucatu, IBB, Department of Botany, 18618-970, PO Box 510, Botucatu city, São Paulo State, Brazil

\section{A R T I C L E I N F O}

\section{Article history:}

Received 19 May 2016

Received in revised form

28 September 2016

Accepted 22 October 2016

Edited by Hermann Heilmeier.

Available online 25 October 2016

\section{Keywords:}

Bundle sheath extension

Leaf anatomy

Leaf thickness

Plant growth form

Plant habit

Savanna

\begin{abstract}
A B S T R A C T
Plant species can be classified into heterobaric and homobaric, depending on whether their leaves present bundle sheath extensions (BSEs) (heterobaric) or not (homobaric). Incidences of the two leaf types seem to be related to growth environment and the light stratification. The Brazilian Cerrado and seasonal semideciduous forests are contrasting environments mainly with regard to irradiance and air humidity. However, studies comparing the distributions of homobaric and heterobaric species in these vegetation types are lacking. We investigate the presence/absence of leaf BSEs across diverse habits (herbs, shrubs and trees) in a seasonal semideciduous forest, cerrado sensu stricto (open physiognomy) and cerradão (forest physiognomy) to evaluate the influence of plant habit and growth environment on the distribution of homobaric and heterobaric species. Leaves from 131 species in 54 angiosperm families were analysed using standard methods of light microscopy. The distribution of the different leaf types in each environment was analysed using the $\chi^{2}$ test and Fisher's exact test $(P<0.05)$. Homobaric and heterobaric leafed species occur in all environments and do not correlate with plant habit. Of the total number of species examined, 103 (78.6\%) exhibited homobaric leaves and 28 (21.4\%) presented heterobaric leaves. Of the total of heterobaric species, $8.8 \%$ occurred in the seasonal semideciduous forest, $50 \%$ in the cerradão and $41.2 \%$ in the cerrado sensu stricto. This model of distribution may be related to the higher irradiances in Cerrado. Homobaric leaves of shrubs and trees were thicker in all environments, while heterobaric leaves of herbs were thicker in the Cerrado. This study finds a tendency for the distribution pattern of homobaric or heterobaric leaves to reflect both the growth environment and the taxonomic grouping. Since leaf type is a constitutive characteristic, interspecific differences in leaf type may reflect particular physiological behaviours, linking them to success in colonising particular environments.
\end{abstract}

(c) 2016 Elsevier GmbH. All rights reserved.

\section{Introduction}

Based on the presence or absence of bundle-sheath extensions (BSEs) in their leaves, plant species are generally classified into two groups - those having heterobaric leaves and those having homobaric leaves. In a heterobaric leaf, the parenchyma or sclerenchyma cells of the BSE extend to the epidermises on each side of the leaf (Kenzo et al., 2007), effectively isolating the leaf airspaces into numerous compartments (Terashima, 1992). In contrast, the BSEs are lacking in homobaric leaves, so the internal airspaces are essentially continuous (Kenzo et al., 2007).

The presence/absence of BSEs affects both the mechanical and the physiological properties of the two leaf types (Kenzo et al.,

\footnotetext{
* Corresponding author.

E-mail address: tatiane@ibb.unesp.br (T.M. Rodrigues).
}

2007; Liakoura et al., 2009; Inoue et al., 2015). In homobaric leaves, the structural continuity of the leaf allows more efficient gas diffusion (Rhizopoulou and Psaras, 2003). On the other hand, the BSEs of heterobaric leaves may act as water (Wylie, 1943) and light conduits (Karabourniotis et al., 2000) to the inner layers of the mesophyll and may also give additional mechanical support to the leaf blade (Esau, 1977). In addition, higher rates of photosynthesis and transpiration have been recorded in heterobaric species (Inoue et al., 2015). Therefore, the architectural arrangement of a leaf's tissues may influence its physiological performance (Reich et al., 2003). This adaptation has been considered important in terms of specific growth environments and/or life-form types (Kenzo et al., 2007; Rossato et al., 2015). In addition, studies indicate that homobaric leaves are thicker than heterobaric leaves (Boeger et al., 2016) and present a higher proportion of photosynthetic areas in the mesophyll which is advantageous for capturing diffuse light (Terashima, 1992). 
Table 1

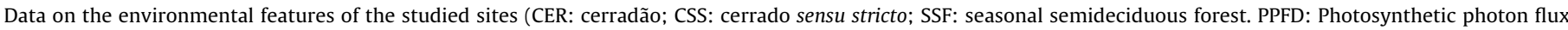
density).

\begin{tabular}{|c|c|c|c|}
\hline \multirow[t]{2}{*}{ Environmental characteristics } & \multicolumn{3}{|l|}{ Studied sites } \\
\hline & CER & CSS & SSF \\
\hline Average daily PPFD $\left(\mu \mathrm{mol} \mathrm{m}^{-2} \mathrm{~s}^{-1}\right)$ & 437 & 804 & 337 \\
\hline Maximum - average - minimum daily relative humidity of air (\%) & $52-41-30$ & $49-39-28$ & $72-69-65$ \\
\hline Average annual precipitation $(\mathrm{mm})$ & 1454 & 1454 & 1428 \\
\hline Length of dry season (months) & 5 & 5 & 5 \\
\hline Average altitude $(\mathrm{m})$ & 720 & 720 & 786 \\
\hline Minimun - average - maximun temperature $\left({ }^{\circ} \mathrm{C}\right)$ & $12.5-20.8-29$ & $13.4-21.7-30$ & $12-20.3-28.5$ \\
\hline Soil type & latosols and argisols & latosols and argisols & podzolics and litholics \\
\hline
\end{tabular}

Recent studies have sought to correlate leaf type with environment, with life-form type and with plant habit (Kenzo et al., 2016). Thus, some researchers have shown that heterobaric species are more common in dry and/or cold deciduous forests (Terashima, 1992), while homobaric species predominate in the evergreen forests of humid and warm climates (Boeger et al., 2004; Kenzo et al., 2007). However, the distribution of light intensity, temperature and humidity is variable even inside a tropical evergreen forest (Whitmore, 1988). In this sense, Kenzo et al. (2007) proposed that the leaf type seems also to be correlated to the life form type of the species in a tropical forest, i.e. emergent, canopy, understorey, or canopy-gap species. Species in the upper canopy and in canopy gaps are exposed to higher irradiance and lower humidity than species in the understorey (Whitmore, 1988) favouring the abundance of heterobaric leafed species in these higher canopy layers and in gaps (Kenzo et al., 2007). Moreover, heterobaric leaves seem to be more common among tree and shrub species than among herbs in xeric environments (Liakoura et al., 2009).

The Brazilian savanna (local name Cerrado) and seasonal semideciduous forests present contrasting environmental features, mainly regarding irradiance (Hoffman and Franco, 2003; Tresmondi et al., 2015). The Cerrado is a vegetation mosaic composed of different physiognomies varying from open vegetation with continuous herbaceous and sparse woody plants (cerrado sensu stricto) to a true forest formation (cerradão). This ecosystem is characterised by a strongly seasonal climate with distinctive rainy summers and dry winters. The soils are deep and well drained, acidic, extremely low in available nutrients and with high aluminium contents (Oliveira and Marquis, 2002). The seasonal semideciduous forest is characterised by dense vegetation with a closed canopy and a layered structure, with trees up to $30 \mathrm{~m}$ high. The species are conditioned to eutrophic soils (Scariot and Sevilha, 2005) and seasonal climates that determine the semideciduous nature of the forest canopy. In this sense, we hypothesise that the environmental conditions of the Cerrado could favour the higher abundance of heterobaric leafed species. However, studies comparing the distribution of homobaric and heterobaric leaves in the Cerrado and forest are lacking.

Here, we investigate the presence/absence of leaf BSEs across diverse habits (herbs, shrubs and trees) and different vegetation types (seasonal semideciduous forest, cerrado sensu stricto and cerradão) in order to evaluate the influence of plant habit and growth environment on the distribution of homobaric and heterobaric leafed species. In addition, we measured the blade thickness looking for a relation of this anatomical trait with the leaf type.

\section{Material and methods}

\subsection{Areas of study and plant material}

This study was conducted during 2014 and 2015 in two contiguous Cerrado physiognomies (cerradão and cerrado sensu stricto) located in Palmeira da Serra farm, Pratânia municipality $\left(22^{\circ} 48^{\prime}\right.$ $20^{\prime \prime} \mathrm{S}, 48^{\circ} 44^{\prime} 36^{\prime \prime} \mathrm{W}$ ) and in a remnant area of seasonal semideciduous forest located in Edgardia farm, in Botucatu municipality $\left(22^{\circ}\right.$ $52^{\prime} \mathrm{S}, 48^{\circ} 26^{\prime} \mathrm{W}$ ), both in central-west region of São Paulo State, in southeastern Brazil. Pratânia and Botucatu municipalities are $37 \mathrm{~km}$ distant from each other. The south marginal areas of the Cerrado and the seasonal semideciduous forest are both characterised by marked climatic seasonality (Tresmondi et al., 2015). The climate in these areas is Cfa according to Köppen classification, that is, temperate (mesothermic) climate, typical of constantly humid region (Setzer, 1966), with rains in the summer and drought in the winter, and with small hydric deficiency from April to August (Cunha and Martins, 2009).

The environmental characterization of each area is summarized in Table 1.

The cerradão (Fig. 1a) and cerrado sensu stricto (Fig. 1b) are contiguous areas and encompass a total of 180 ha. In the Cerrado area, 120 species of angiosperms belonging to 52 families were investigated in the cerradão and 168 species belonging to 51 families in cerrado sensu stricto (Ishara, 2010). The studied forest fragment (Fig. 1c) was 56 ha in size, where 61 tree species belonging to 31 angiosperm families were inventoried (Fonseca and Rodrigues, 2000).

Based on previous phytosociological surveys (Fonseca and Rodrigues, 2000; Ishara et al., 2008; Carvalho et al., 2010; Jorge et al., 2015) a square of $400 \mathrm{~m}^{2}$ was selected inside cerradão, cerrado sensu stricto and forest that encompassed a representative portion of the vegetation with heterogeneity of species and habits. Ten fully-expanded and non-senescent leaves were collected from each of the plants enclosed, in the rainy season (December 2014 to February 2015). In all, 51 species were collected in the cerradão (27 trees, 17 shrubs and 7 herbs), 55 species in the cerrado sensu stricto (16 trees, 24 shrubs and 15 herbs) and 49 species in the seasonal semideciduous forest ( 22 trees, 17 shrubs and 10 herbs). A total of 131 species belonging to 54 angiosperm families were examined. Since some species occurred in two or more environments, the total number of plants studied was 155 (Table 2).

Vouchers were incorporated in the collection of Herbarium Irina Delanova de Gemtchujnicov (BOTU), IBB UNESP, Botucatu/SP.

\subsection{Light microscopy}

For anatomical analysis, samples excised from the median region of the leaf blade were fixed in FAA 50 (Johansen, 1940) and processed according to two protocols. Most samples were crosssectioned (12 $\mu \mathrm{m}$ thickness) using a Ranvier microtome, stained with safranine and astra blue (Bukatsch, 1972) and mounted in glycerine jelly. Others were dehydrated in an alcohol series and embedded in methacrylate resin, cross sectioned $(5 \mu \mathrm{m}$ in thickness) using a rotatory microtome and stained with toluidine blue $\mathrm{O}$ $0.05 \%$ pH 4.7 (O'Brien et al., 1964). Permanent slides were mounted using synthetic resin.

The slides were examined under a light microscope (Olympus BX 41) equipped with a digital camera. The leaf thickness was cal- 
Table 2

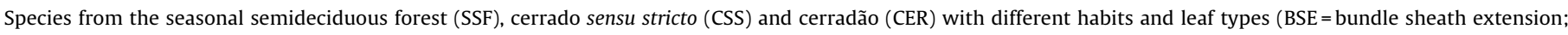
$\mathrm{ES}=$ esclerenchyma; PA: parenchyma).

\begin{tabular}{|c|c|c|c|c|c|c|}
\hline Family & Species & Plant habit & $\begin{array}{l}\text { Vegetation } \\
\text { type }\end{array}$ & Leaf type & $\begin{array}{l}\text { BSE cell } \\
\text { type }\end{array}$ & $\begin{array}{l}\text { Average of leaf } \\
\text { thickness }(\mu \mathrm{m})\end{array}$ \\
\hline \multirow[t]{3}{*}{ Acanthaceae } & Ruellia sp. & herb & SSF & Homobaric & - & 124.65 \\
\hline & Justicia brasiliana Roth & shrub & SSF & Homobaric & - & 97.89 \\
\hline & Justicia sp. & shrub & SSF & Homobaric & - & 100.60 \\
\hline Amaranthaceae & Gomphrena macrocephala A.St.-Hil. & herb & CSS & Homobaric & - & 203.27 \\
\hline \multirow[t]{3}{*}{ Anacardiaceae } & Anacardium humile A.St.-Hil. & shrub & CSS & Heterobaric & ES & 189.13 \\
\hline & Astronium graveolens Jacq. & tree & SSF & Homobaric & - & 140.69 \\
\hline & Lithraea molleoides (Vell.) Engl. & tree & CER & Homobaric & - & 277.75 \\
\hline \multirow[t]{3}{*}{ Annonaceae } & Annona sp. & shrub & CSS & Homobaric & - & 195.70 \\
\hline & Annona coriacea Mart. & shrub & CSS, CER & Homobaric & - & $207.37,195.46$ \\
\hline & Duguetia furfuracea (A.St.-Hil.) Benth. \& Hook. & tree & CSS & Heterobaric & ES & 93.16 \\
\hline \multirow[t]{2}{*}{ Apocynaceae } & Aspidosperma polyneuron Müll. Arg. & tree & SSF & Homobaric & - & 145.27 \\
\hline & Aspidosperma ramiflorum Müll. Arg. & shrub & SSF & Homobaric & - & 114.15 \\
\hline \multirow[t]{6}{*}{ Asteraceae } & Chaptalia sp. & herb & CSS & Homobaric & - & 132.67 \\
\hline & Acanthospermum australe (Loefl.) Kuntze & herb & CSS & Homobaric & - & 97.62 \\
\hline & Baccharis dracunculifolia DC. & shrub & CSS & Homobaric & - & 134.31 \\
\hline & Emilia sonchifolia (L.) DC. Ex Wight & herb & CSS & Homobaric & - & 134.84 \\
\hline & Gochnatia pulchra Cabrera & shrub & CSS, CER & Heterobaric & ES & $120.66,130.02$ \\
\hline & Moquiniastrum barrosoae (Cabrera) G. Sancho & shrub & CSS, CER & Heterobaric & ES & $72.38,69.96$ \\
\hline \multirow{3}{*}{ Bignoniaceae } & Fridericia sp. & shrub & CSS & Homobaric & - & 80.79 \\
\hline & Handroanthus serratifolius (Vahl.) S. Grose & tree & CSS, CER & Heterobaric & ES & $160.15,138.14$ \\
\hline & Zeyheria montana Mart. & tree & CSS, CER & Homobaric & - & $100.25,99.84$ \\
\hline Boraginaceae & Heliotropium sp. & herb & SSF & Homobaric & - & 108.39 \\
\hline \multirow[t]{3}{*}{ Bromeliaceae } & Ananas ananassoides (Baker) L.B.Sm. & herb & CSS & Homobaric & - & 206.87 \\
\hline & Bromelia balansae Mez & herb & CSS, CER & Homobaric & - & $115.68,112.92$ \\
\hline & Bromelia sp. & shrub & CSS & Homobaric & - & 271.55 \\
\hline Cannabaceae & Celtis fluminensis Carauta & shrub & SSF & Homobaric & - & 91.51 \\
\hline Caryocaraceae & Caryocar brasiliense Cambess & shrub & CSS & Homobaric & - & 136.61 \\
\hline Celastraceae & Maytenus aquifolium Mart. & shrub & SSF & Homobaric & - & 100.54 \\
\hline \multirow[t]{2}{*}{ Commelinaceae } & Commelina sp.1 & herb & SSF & Homobaric & - & 139.32 \\
\hline & Commelina sp. 2 & herb & CER & Homobaric & - & 141.77 \\
\hline Convolvulaceae & Evolvulus sp. & herb & CSS & Homobaric & - & 62.39 \\
\hline Cucurbitaceae & Cayaponia espelina (Silva Manso) Cogn. & herb & CSS & Heterobaric & ES & 215.55 \\
\hline Cyperaceae & Hypolytrum pungens (Vahl.) Kunth & herb & CER & Heterobaric & ES & 214.61 \\
\hline Dilleniaceae & Davilla sp. & shrub & CSS & Homobaric & - & 189.71 \\
\hline \multirow{2}{*}{ Erythroxylaceae } & Erythroxylum campestre A.St.-Hil. & shrub & CSS & Homobaric & - & 144.03 \\
\hline & Erythroxylum deciduum A.St.-Hil. & shrub & CER & Homobaric & - & 88.97 \\
\hline Euphorbiaceae & Acalypha sp. & herb & SSF & Homobaric & - & 110.80 \\
\hline & Chamaesyce Gray & herb & CSS & Homobaric & - & 129.18 \\
\hline & Actinostemon concepcionis (Chodat \& Hassl.) Hochr. & shrub & SSF & Homobaric & - & 78.36 \\
\hline & Croton floribundus Spreng. & tree & SSF & Heterobaric & PA & 130.01 \\
\hline & Croton glandulosus L. & herb & CSS & Homobaric & - & 139.24 \\
\hline Fabaceae & Andira humilis Mart. ex. Benth. & shrub & CSS, CER & Heterobaric & ES & $185.53,131.35$ \\
\hline & Bauhinia sp. & shrub & CSS & Heterobaric & ES & 86.94 \\
\hline & Bauhinia longifolia (Bong.) Steud. & shrub & SSF & Heterobaric & ES & 78.59 \\
\hline & Bauhinia rufa (Bong.) Steud. & shrub & CSS, CER & Heterobaric & ES & $98.31,90.92$ \\
\hline & Bowdichia virgiloides Kunth & tree & CER & Homobaric & - & 105.16 \\
\hline & Chamaecrista flexuosa (L.) Greene & shrub & CSS & Homobaric & - & 106.01 \\
\hline & Copaifera langsdorffii Desf. & tree & CER & Heterobaric & ES & 190.81 \\
\hline & Dimorphandra mollis Benth. & tree & CSS, CER & Homobaric & - & $128.64,126.05$ \\
\hline & Holocalyx balansae Micheli & tree & SSF & Homobaric & - & 120.55 \\
\hline & Inga striata Benth. & tree & SSF & Homobaric & - & 110.61 \\
\hline & Machaerium sp. Pers. & tree & CER & Heterobaric & PA & 199.83 \\
\hline & Peltophorum dubium (Spreng.) Taub. & tree & SSF & Homobaric & - & 81.82 \\
\hline & Platypodium elegans Vogel & tree & SSF, CER & Homobaric & - & $211.09,190.89$ \\
\hline & Senna rugosa (G. Don) H.S.Irwin \& Barneby & shrub & CSS, CER & Homobaric & - & $95.12,90.65$ \\
\hline & Stryphnodendron adstringens (Mart.) Coville & tree & CSS & Homobaric & - & 124.29 \\
\hline Lacistemataceae & Lacistema hasslerianum Chodat & shrub & CER & Homobaric & - & 100.39 \\
\hline Lamiaceae & Eriope crassipes Benth. & herb & CSS & Heterobaric & PA & 84.96 \\
\hline & Aegiphila sellowiana Cham. & tree & CER & Homobaric & - & 180.61 \\
\hline & Hyptis sp. & herb & CSS & Homobaric & - & 98.05 \\
\hline Lecythidaceae & Cariniana estrellensis (Raddi) Kuntze & tree & SSF & Homobaric & - & 102.33 \\
\hline Loganiaceae & Strychnos brasiliensis Mart. & shrub & SSF & Homobaric & - & 135.21 \\
\hline Lythraceae & Cuphea calophylla Cham. \& Schltdl. & herb & SSF & Homobaric & - & 71.00 \\
\hline Malpighiaceae & Byrsonima pachyphylla A. Juss. & shrub & CER & Homobaric & - & 200.99 \\
\hline & Heteropterys sp. & shrub & CSS & Heterobaric & ES & 163.57 \\
\hline Malvaceae & Eriotheca grassilipes Schott \& Endl. & tree & CSS & Heterobaric & ES & 177.15 \\
\hline & Luehea grandiflora Mart. \& Zucc. & tree & CER & Heterobaric & PA & 61.83 \\
\hline & Pseudobombax longiflorum (Mart. et Zucc.) A. Robyns & tree & CER & Heterobaric & ES & 131.90 \\
\hline & Sida sp. & herb & CSS & Homobaric & - & 95.63 \\
\hline & Waltheria communis A.St-Hill & shrub & CSS & Heterobaric & ES & 143.55 \\
\hline Melastomataceae & Miconia albicans (Sw.) Triana & shrub & CSS, CER & Homobaric & - & $161.46,156.59$ \\
\hline & Miconia langsdorffii Cong. & shrub & CER & Homobaric & - & 150.11 \\
\hline
\end{tabular}


Table 2 (Continued)

\begin{tabular}{|c|c|c|c|c|c|c|}
\hline Family & Species & Plant habit & $\begin{array}{l}\text { Vegetation } \\
\text { type }\end{array}$ & Leaf type & $\begin{array}{l}\text { BSE cell } \\
\text { type }\end{array}$ & $\begin{array}{l}\text { Average of leaf } \\
\text { thickness }(\mu \mathrm{m})\end{array}$ \\
\hline & Miconia fallax DC. & tree & CSS & Homobaric & - & 142.59 \\
\hline \multirow[t]{4}{*}{ Meliaceae } & Trichilia casaretti C.DC. & tree & SSF & Homobaric & - & 110.49 \\
\hline & Trichilia catigua A.Juss. & tree & SSF & Homobaric & - & 138.34 \\
\hline & Trichilia elegans A.Juss. & shrub & SSF & Homobaric & - & 105.28 \\
\hline & Trichilia pallida Sw. & tree & SSF & Homobaric & - & 124.13 \\
\hline \multirow[t]{13}{*}{ Myrtaceae } & Calyptranthes lucida Mart. ex. DC. & shrub & CER & Homobaric & - & 160.61 \\
\hline & Eugenia aurata O. Berg & shrub & CER & Homobaric & - & 162.17 \\
\hline & Eugenia blastantha (O.Berg) D. Legrand & tree & SSF & Homobaric & - & 140.78 \\
\hline & Eugenia florida DC. & tree & SSF, CER & Homobaric & - & $150.98,160.56$ \\
\hline & Eugenia sp. & tree & CSS & Homobaric & - & 206.78 \\
\hline & Myrcia sp. 1 & tree & CER & Homobaric & - & 195.73 \\
\hline & Myrcia sp. 2 & tree & CER & Homobaric & - & 194.69 \\
\hline & Myrcia bela Cambess. & tree & CSS, CER & Homobaric & - & $131.91,125.61$ \\
\hline & Myrcia lingua (O.Berg) Mattos & tree & CSS, CER & Homobaric & - & $220.23,212.13$ \\
\hline & Myrcia multiflora (Lam.) DC. & tree & CSS & Homobaric & - & 212.52 \\
\hline & Myrcia selloi (Spreng.) N.Silveira & tree & CER & Homobaric & - & 195.81 \\
\hline & Myrciaria floribunda Miq. & shrub & SSF & Homobaric & - & 92.71 \\
\hline & Neomitranthes glomerata (D.Legrand) D. Legrand & tree & SSF & Homobaric & - & 141.11 \\
\hline Ochnaceae & Ouratea spectabilis (Mart.) Engl. & tree & CSS, CER & Homobaric & - & $88.23,85.42$ \\
\hline Opiliaceae & Agonandra engleri Hoehne & tree & SSF & Homobaric & - & 118.81 \\
\hline Orchidaceae & Oeceoclades maculata (Lindl.) Lindl. & herb & SSF, CER & Homobaric & - & $280.28,250.61$ \\
\hline Oxalidaceae & Oxalis sp. & herb & SSF & Homobaric & - & 100.03 \\
\hline Passifloraceae & Piriqueta Aubl. & herb & CSS & Homobaric & - & 107.66 \\
\hline Peraceae & Pera glabrata (Schott) Poepp. ex Baill. & tree & CSS, CER & Homobaric & - & $143.09,141.08$ \\
\hline Phytolaccaceae & Gallesia integrifolia (Spreng.) Harms & tree & SSF & Homobaric & - & 157.53 \\
\hline \multirow[t]{2}{*}{ Piperaceae } & Piper amalago $\mathrm{L}$. & shrub & SSF & Homobaric & - & 140.76 \\
\hline & Piper arboreum Aubl. & shrub & SSF & Homobaric & - & 184.66 \\
\hline \multirow[t]{2}{*}{ Poaceae } & Panicum sp. 1 & herb & SSF, CER & Heterobaric & ES & $94.10,100.69$ \\
\hline & Panicum sp. 2 & herb & CER & Homobaric & - & 102.54 \\
\hline \multirow[t]{2}{*}{ Portulacaceae } & Talinum paniculatum (Jacq.) Gaertn. & herb & SSF & Homobaric & - & 98.99 \\
\hline & Portulaca oleracea L. & herb & SSF & Homobaric & - & 250.50 \\
\hline \multirow[t]{2}{*}{ Primulaceae } & Myrsine guianensis (Aubl.) Kuntze & tree & CSS, CER & Homobaric & - & $224.58,215.24$ \\
\hline & Myrsine umbellata Mart. & tree & SSF, CER & Homobaric & - & $163.46,170.09$ \\
\hline Proteaceae & Roupala montana Aubl. & tree & CER & Heterobaric & ES & 231.81 \\
\hline Rhamnaceae & Rhamnidium elaeocarpum Reissek & tree & SSF & Homobaric & - & 95.79 \\
\hline \multirow[t]{5}{*}{ Rubiaceae } & Cordiera sessilis (Vell.) Kuntze & shrub & CER & Homobaric & - & 170.51 \\
\hline & Borreria schumannii (Standl. ex Bacigalupo) E.L.Cabral \& Sobrado & herb & CSS & Homobaric & - & 98.37 \\
\hline & Psychotria carthagenensis Jacq. & shrub & SSF & Homobaric & - & 117.60 \\
\hline & Psychotria sp. & herb & CER & Homobaric & - & 99.13 \\
\hline & Tocoyena formosa (Cham. \& Schltdl.) K.Schum. & shrub & CSS & Homobaric & - & 78.55 \\
\hline \multirow[t]{2}{*}{ Rutaceae } & Metrodorea nigra A.St.-Hil. & tree & SSF & Homobaric & - & 187.82 \\
\hline & Zanthoxylum fagara (L.) Sarg. & tree & CSS & Homobaric & - & 140.12 \\
\hline Salicaceae & Casearia sylvestris Sw. & tree & SSF, CSS, CER & Homobaric & - & $120.44,190.35,130.78$ \\
\hline Sapotaceae & Chrysophyllum gonocarpum (Mart. \& Eichler ex Miq.) Engl. & tree & SSF & Homobaric & - & 117.80 \\
\hline Siparunaceae & Siparuna guianensis Aubl. & shrub & CER & Homobaric & - & 102.81 \\
\hline \multirow[t]{4}{*}{ Solanaceae } & Cestrum mariquitensis Kunth. & shrub & SSF & Homobaric & - & 76.31 \\
\hline & Solanum sp. 1 & herb & CSS & Homobaric & - & 128.14 \\
\hline & Solanum sp. 2 & herb & CSS & Homobaric & - & 128.00 \\
\hline & Solanum lycocarpum A.St.-Hil & shrub & CER & Heterobaric & ES & 121.46 \\
\hline Thymelaeaceae & Daphnopsis utilis Warms & tree & CER & Homobaric & - & 160.54 \\
\hline Verbenaceae & Lantana camara L. & shrub & CSS, CER & Heterobaric & PA & $172.88,170.03$ \\
\hline \multirow[t]{2}{*}{ Violaceae } & Hybanthus atropurpureus (A.St.-Hil) Taub. & shrub & SSF & Homobaric & - & 86.11 \\
\hline & Hybanthus sp. & shrub & SSF & Homobaric & - & 70.17 \\
\hline Vitaceae & Cissus sp. & shrub & CSS & Homobaric & - & 106.55 \\
\hline \multirow[t]{3}{*}{ Vochysiaceae } & Qualea dichotoma (Mart.) Warm. & tree & CER & Heterobaric & PA & 110.09 \\
\hline & Qualea grandiflora Mart. & tree & CER & Heterobaric & ES & 114.06 \\
\hline & Vochysia tucanorum Mart. & tree & CER & Heterobaric & PA & 112.48 \\
\hline
\end{tabular}

culated in cross sections of 5 individuals of each species $(n=5)$ using the software Olympus Cell B.

For analyses of the leaf surfaces, fresh samples were analysed under a stereomicroscope Leica M205C.

\subsection{Statistical analyses}

The frequency of homobaric and heterobaric leafed species among the vegetation types and the plant habits was compared using the $\chi 2$ test and the Fisher exact test with 5\% significance level when the frequency was lower than five (Snedecor and Cochran, 1974), using SAS 9.2 software. The leaf thickness in homobaric and heterobaric leaves in each environment and habitat was subjected to Levene's test to determine the homogeneity of the treatment variances. Data were subjected to an analysis of variance (ANOVA), and the means were compared by Tukey's test with a $5 \%$ probability $(P \leq 0.05)$.

\section{Results}

Both homobaric (Fig. 2a) and heterobaric (Fig. 2b,c) leafed species occurred in the seasonal semideciduous forest, in the cerradão and in the cerrado sensu stricto (Table 2). The homobaric species predominated in all vegetation types (Table 3 ). Of the 131 species studied, 103 (78.6\%) exhibited homobaric leaves.

The proportion of species with each leaf type differed among the study areas ( $\chi^{2}$ test, $P \leq 0.05, \mathrm{df}=2, N=155$; Table 3 ). In the seasonal semideciduous forest, $6.1 \%$ of the species presented heterobaric leaves, while in the cerradão and the cerrado sensu stricto the heterobaric species represented were 33.3 and $25.5 \%$, respec- 
Table 3

Number of species with heterobaric and homobaric leaves in the seasonal semideciduous forest, cerradão and cerrado sensu stricto $\left(\chi^{2}\right.$ test, $P \leq 0.05$, df $=2, N=155$ ).

\begin{tabular}{|c|c|c|c|}
\hline Vegetation type & Total of sampled species & Species with heterobaric leaves & Species with homobaric leaves \\
\hline Seasonal semideciduous forest & 49 & 3 & 46 \\
\hline Cerradão & 51 & 17 & 34 \\
\hline Cerrado sensu stricto & 55 & 14 & 41 \\
\hline
\end{tabular}

Table 4

Number of heterobaric and homobaric leafed species grouped according to their different habits.

\begin{tabular}{|c|c|c|c|}
\hline Plant habit & Total of sampled species & Species with heterobaric leaves & Species with homobaric leaves \\
\hline Herbs & 10 & 1 & 9 \\
\hline Shrubs & 17 & 1 & 16 \\
\hline Trees & 22 & 1 & 21 \\
\hline
\end{tabular}

b) in the cerrado sensu stricto (Fisher exact test, $P \leq 0.05, \mathrm{df}=2, N=55$ ).

\begin{tabular}{|c|c|c|c|}
\hline Plant habit & Total of sampled species & Species with heterobaric leaves & Species with homobaric leaves \\
\hline Herbs & 15 & 2 & 13 \\
\hline Shrubs & 24 & 9 & 15 \\
\hline Trees & 16 & 3 & 13 \\
\hline
\end{tabular}

c) in the cerradão (Fisher exact test, $P \leq 0.05, \mathrm{df}=2, N=51$ ).

\begin{tabular}{|c|c|c|c|}
\hline Plant habit & Total of sampled species & Species with heterobaric leaves & Species with homobaric leaves \\
\hline Herbs & 7 & 2 & 5 \\
\hline Shrubs & 17 & 6 & 11 \\
\hline Trees & 27 & 9 & 18 \\
\hline
\end{tabular}

tively. Of the 34 heterobaric leafed species, 3 (9\%) occurred in the seasonal semideciduous forest, 17 (50\%) in the cerradão, and 14 (41\%) in the cerrado sensu stricto.

Differences in the distributions of homobaric and heterobaric species with the three habits were not significant in any of the areas studied (Fisher exact test, $P \geq 0.05, \mathrm{df}=2, N=49$ for seasonal semideciduous forest, Table 4a; Fisher exact test, $P \geq 0.05, \mathrm{df}=2$, $N=55$ for cerrado sensu stricto, Table $4 \mathrm{~b}$; and Fisher exact test, $P \geq 0.05, \mathrm{df}=2, N=51$ for cerradão, Table $4 \mathrm{c}$ ). However, there may be a tendency for a greater abundance of heterobaric species among trees and shrubs in the Cerrado. In the cerrado sensu stricto, $64.3 \%$ of the heterobaric leafed species were shrubby (Table 4b) and in the cerradão, $35 \%$ of them were shrubs and $52.9 \%$ trees (Table $4 c$ ). In the seasonal semideciduous forest, the percentages of species with heterobaric leaves were about the same among herbs, shrubs and trees (Table 4a).

Concerning the cell composition, heterobaric leaves can present parenchymatous or sclerenchymatous BSE. In seasonal semideciduous forest, $66.7 \%$ of the heterobaric leafed species presented sclerenchymatous BSE and 33.3\% had parenchymatous BSE. In cerradão, $70.6 \%$ of the sampled heterobaric leafed species presented sclerenchymatous BSE and $29.4 \%$ had parenchymatous BSE. In the cerrado sensu stricto, $85.8 \%$ of the heterobaric leafed species exhibited sclerenchymatous BSE and $23.5 \%$ presented parenchymatous BSE. Concerning the distribution of sclerenchymatous and parenchymatous BSE among the different studied families, we observed that three plant families presented only species with parenchymatous BSE; 10 families exhibited species with sclerenchymatous BSE and four families presented both parenchymatous and sclerenchymatous BSE (Table 2).

The comparison of homobaric and heterobaric leaves showed that homobaric leaves of shrubs and trees were thicker in all environments, while heterobaric leaves of herbs are thicker in the Cerrado (Table 5).
Table 5

Average thickness $(\mu \mathrm{m})$ of homobaric and heterobaric leaves by environment and plant habit (CER = cerradão, $\mathrm{CSS}=$ cerrado sensu stricto, $\mathrm{SSF}=$ seasonal semideciduous forest). Values followed by the same letter do not differ significantly according to Tukey's test $(P<0.0001, n=5)$; lower case letters in the column and capital letters in the line.

\begin{tabular}{llll}
\hline Leaf type & \multicolumn{3}{l}{ Environment } \\
\cline { 2 - 4 } & CER & CSS & SSF \\
\hline Herb & & \\
Heterobaric & $157.66 \mathrm{aA}$ & $150.26 \mathrm{aB}$ & $94.10 \mathrm{bC}$ \\
Homobaric & $141.40 \mathrm{bA}$ & $130.14 \mathrm{bB}$ & $130.93 \mathrm{aB}$ \\
& Shrub & & \\
Heterobaric & $117.40 \mathrm{bB}$ & $138.04 \mathrm{bA}$ & $78.59 \mathrm{bC}$ \\
Homobaric & $137.87 \mathrm{aB}$ & $142.01 \mathrm{aA}$ & $110.96 \mathrm{aC}$ \\
Heterobaric & Tree & & \\
Homobaric & $143.44 \mathrm{bA}$ & $143.49 \mathrm{bA}$ & $130.02 \mathrm{bB}$ \\
C. V. $=34 \%$ & $166.79 \mathrm{aA}$ & $153.49 \mathrm{aB}$ & $134.86 \mathrm{aC}$ \\
\hline
\end{tabular}

\section{Discussion}

The occurrence of homobaric and heterobaric leafed species did not depend on the habit types or on the vegetation formations. Homobaric and heterobaric leaves occurred in herbaceous, shrubby and tree species growing in the forest and also in the savanna ecosystems (both the cerradão and the cerrado sensu stricto).

A predominance of homobaric leafed species is commonly associated with forest trees of warm, humid regions (Wylie, 1952; McClendon, 1992; Kashimura et al., 2000; Boeger et al., 2004; Kenzo et al., 2007). However, our results show that homobaric leafed species also predominate in forest and savanna systems, having contrasting environments mainly with regard to irradiance (Hoffman and Franco, 2003; Tresmondi et al., 2015). According to Kenzo et al. (2007), shade and wet conditions are more suited to homobaric leaves than to heterobaric leaves. The predominance of homobaric leafed species in the cerrado sensu stricto is unexpected since this ecosystem is characterised by intense sunlight that generates a drier microclimate (Coutinho, 1978, 2002) compared with either the seasonal semideciduous forest or the cerradão 

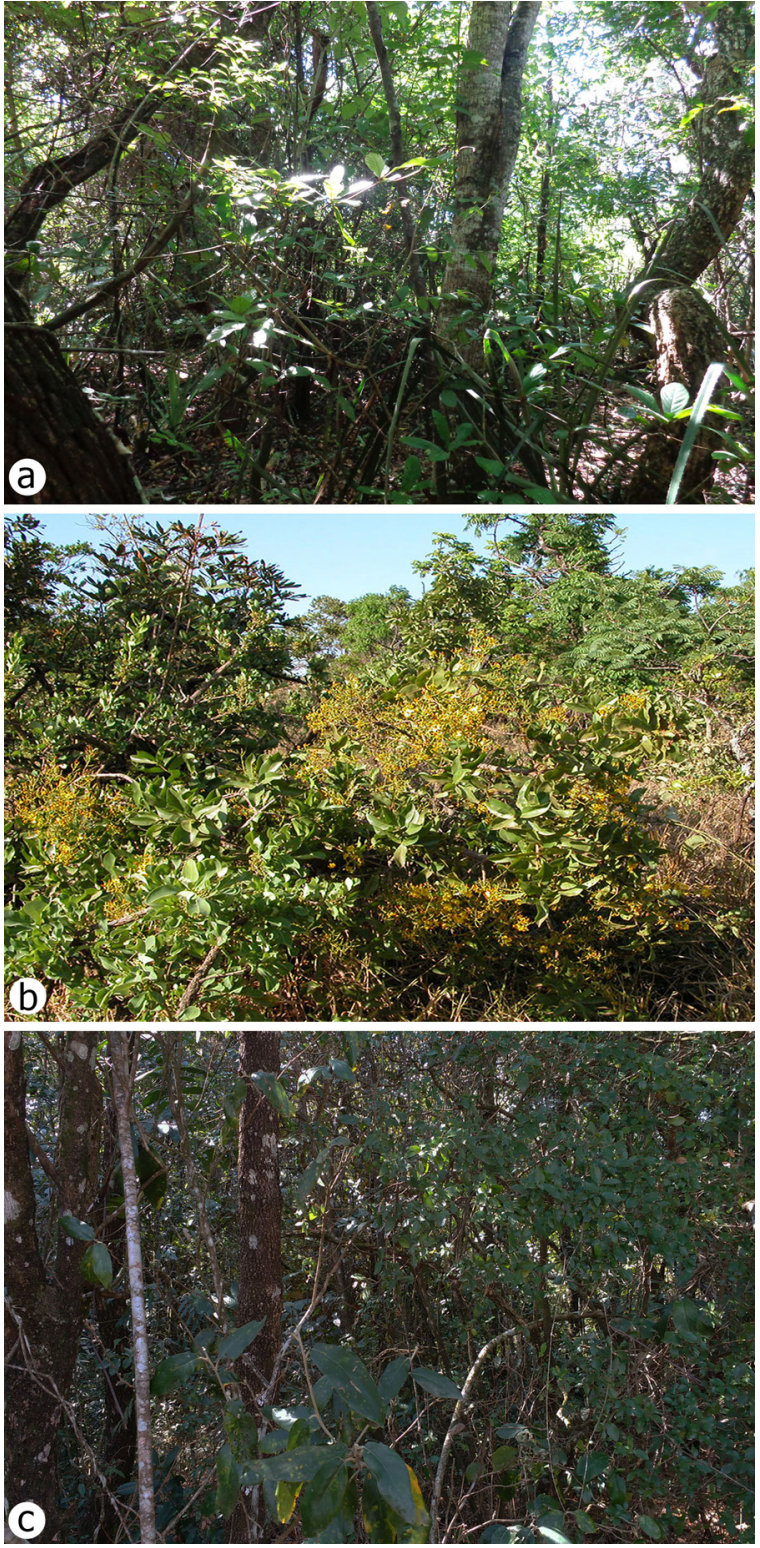

Fig. 1. Aspects of the study areas. A. Cerradão. B. Cerrado sensu stricto. C. Seasonal semideciduous forest.

(Tresmondi et al., 2015). On the other hand, this is not entirely surprisingly, given that shrubs and trees from the Cerrado usually have deep roots and so likely experience satisfactory access to water (Machado et al., 1997).

Nevertheless, comparing the distributions of heterobaric species in the three vegetation types studied, we note that this leaf type is more common in the Cerrado than in the seasonal semideciduous forest. This may be related to the microclimate around the Cerrado plants, i.e. higher irradiance and lower humidity (Tresmondi et al., 2015). Following Kenzo et al. (2007), the compartmentalisation of the leaf mesophyll by BSEs is more common in environments with characteristics that expose the plants to greater levels of desiccation. So, in the Cerrado, the BSEs could be related to the efficient use of water, since they are responsible for the more rapid response of stomata to drought signals such as the reduction of water potential in the mesophyll (Terashima, 1992). In addition, the more efficient water transport in heterobaric leaves reduces water usage associated with photosynthesis more than in homobaric leaves (Inoue et al., 2015). Other roles have been
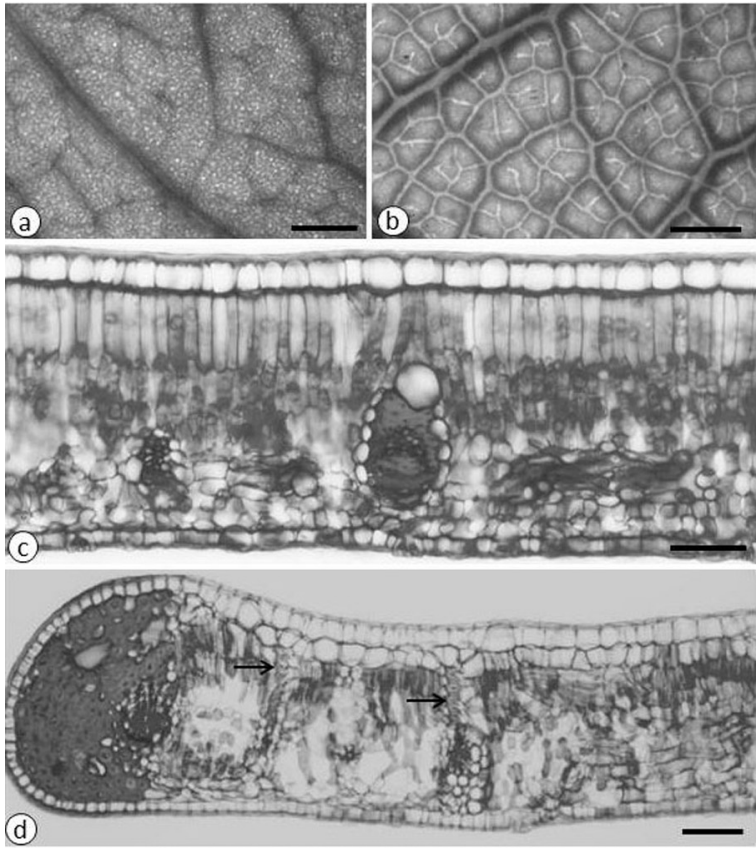

(d)

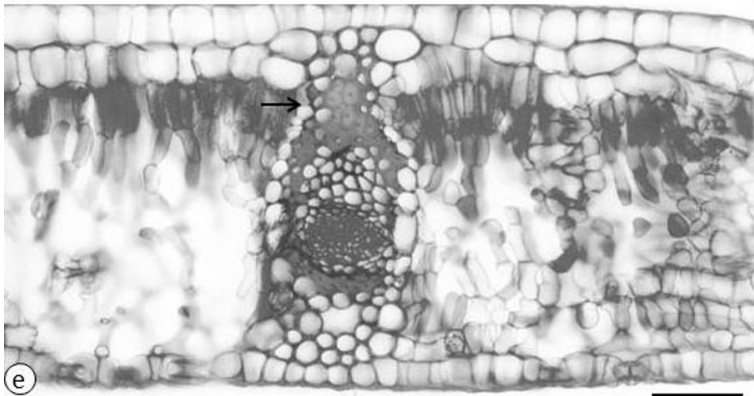

Fig. 2. Light micrographs of representative homobaric and heterobaric leaves. A. The abaxial surface of homobaric leaf of Emilia sonchifolia. B. The abaxial surface of heterobaric leaf of Bauhinia rufa. C. Cross section of homobaric leaf of Cordiera sessilis (Rubiaceae). D, E. Cross sections of heterobaric leaves of Eriotheca grassilipes (Malvaceae). Arrows indicate bundle sheath extensions. Bars: $A, B=500 \mu \mathrm{m}$. C, $\mathrm{D}=150 \mu \mathrm{m}$. $\mathrm{E}=100 \mu \mathrm{m}$.

attributed to BSEs, such as the protection of leaves against water loss after leaf injury (Aldea et al., 2005) and the conduction of visible light to the inner layers of mesophyll in thick leaves (Karabourniotis et al., 2000; Nikolopoulos et al., 2002; Liakoura et al., 2009).

Although we did not observe a direct relation between the leaf type and habit, heterobaric leaves do seem to be more common among shrubs and trees, mainly in the Cerrado. Our results corroborate the findings of McClendon (1992) and Kenzo et al. (2007) who report the frequency of leaves with BSEs is higher among plants in the higher strati of the canopy and also those found in gaps. Clearly, these plants will be more subject to desiccation by exposure to higher VPDs, higher leaf temperatures, higher irradiation levels and stronger winds (Aoki et al., 1978). In this situation, the presence of BSEs could represent an advantage due to their action in the rapid stomatal responses to desiccation and also in the structural protection they offer reducing leaf compression under conditions of low water status as has been suggested by Terashima (1992) and Kenzo et al. (2007). Future research should involve experimental verification that the hypothesised physiological benefits of the heterobaric feature translate to actual physiological benefits in these species.

Sclerenchymatous BSE were more abundant in the three studied environments in comparison with parenchymatous BSE, wherein the higher proportion of species with sclerenchymatous BSE was observed in the Cerrado. This is a xeromorphic aspect that has 
been associated with the environmental conditions of the Cerrado (Bieras and Sajo, 2009; Rossato et al., 2015) and enable the species to cope with abiotic filters such as drought, high light intensities and oligotrophic soils (Rossato et al., 2015) in addition to provide mechanical support and protection against leaf collapse after water deficit (Wylie, 1943; Terashima, 1992; Kenzo et al., 2007) and herbivores (Sack and Scoffoni, 2013).

The highest thickness of the homobaric leaves as in shrub and trees in Cerrado and seasonal forest is a common trait to other studies (Terashima, 1992; Kenzo et al., 2007; Liakoura et al., 2009; Pieruschka et al., 2010; Lynch et al., 2012; Boeger et al., 2016) and has been associated to a well-developed spongy parenchyma (Boeger et al., 2016). According to Vogelman et al. (1996), a thicker spongy parenchyma is advantageous for capturing diffuse light, mainly under limited light conditions. The occurrence of thicker heterobaric leaves in herbs from cerrado sensu stricto and cerradão is noticeable in this study. This fact is probably associated with the presence of xeromorphic features in the herb species in these environments. Succulent plants, such as Bromeliaceae species common in Cerrado (Table 1), are characterised by a well-developed water-storage tissue (Esau, 1977). In addition, the developed sclerenchyma and more strongly differentiated palisade parenchyma collaborate to the increased thickness of the leaves (Esau, 1977). In contrast to other studies, we opted for not exclude these species (with very thick leaves) from our results.

Among the species, genera and families which we sampled we note that, at family level, both homobaric and heterobaric leafed species can sometimes occur in the same families e.g. in Anacardiaceae, Annonaceae, Asteraceae, Euphorbiaceae, Fabaceae, Malpighiaceae, Malvaceae. Nevertheless, the majority of species from any particular family presented only one of these leaf types and this consistency seemed to be independent of habit and vegetation type. Thus, species belonging to the Acanthaceae, Bromeliaceae, Erythroxylaceae, Melastomataceae, Meliaceae, Myrtaceae, Rubiaceae, Rutaceae and Violaceae exhibited only homobaric leaves while, in contrast, species belonging to the Vochysiaceae presented only heterobaric leaves.

At the genus level, the majority of species from any particular genus also present the same leaf type. Thus, the Bauhinia species (Fabaceae), and Qualea species (Vochysiaceae) exhibited only heterobaric leaves, while the Justicia species (Acanthaceae), Miconia species (Melastomataceae), Trichilia species (Meliaceae), Myrsine species (Primulaceae), and Eugenia and Myrcia species (Myrtaceae) exhibited only homobaric leaves. The exception was Solanum (Solanaceae) where among the species appearing in our study, some exhibited homobaric and others heterobaric leaf types (Table 2).

Considering the cell composition of the BSE, except in Bignoniaceae, Fabaceae, Malvaceae and Vochysiaceae that present both parenchymatous and sclerenchymatous BSE, most of the plant families here studied exhibited heterobaric leaves with only one cell type, parenchymatous or sclerenchymatous.

It is fair to conclude that, in most cases, leaf type and cell composition of the BSE is related to taxonomic group (Kenzo et al., 2007) and the trait seems to be phylogenetically conserved. To better evaluate these phylogenetic hypotheses, larger numbers of representative species inside these families are under analysis.

Our findings seem to be consistent with hypotheses that seek to explain the distribution and dominance of some families in a given biome. According to Franco (2002), species from the Amazonian and Atlantic basins have contributed to form the Cerrado flora. In this sense, Veloso (1964) affirmed that the semideciduous seasonal forests and the Cerrado originated from the same floristic trunk, and the dominance of some families in a given area results from the higher ability of these plants to survive under the given edaphic and climatic conditions. It is notable that the forests and Cerrado are found in juxtaposition and in the same general climate (Aoki and Santos, 1979), with differences of microclimate and soil seeming to influence the Cerrado distribution more strongly (Alvim and Araujo, 1952; Alvim, 1954; Arens, 1958; Aoki and Santos, 1979).

In summary, this study finds a tendency for the distribution pattern of homobaric or heterobaric leaf types to reflect both the growth environment and also the taxonomic grouping. Since leaf type is a constitutive characteristic - it does not change with leaf development or among individuals of a same species - interspecific differences in leaf type may reflect particular physiological behaviours, linking them to success in colonising particular environments.

Further analysis on the anatomical organization of heterobaric and homobaric leaves as well as the micromorphology of the leaf surfaces in conjunction with the photosynthetic performance of the species are in course.

\section{Acknowledgments}

The authors thank FAPESP (Fundação de Amparo à Pesquisa do Estado de São Paulo) for financial support for this research (Proc. 2014/12482-2) and for the scholarship to S.C.M. Silva (Proc. 2015/16307-3). Also, S.R. Machado received grants (PQ/Proc. 304396/2015-0) and Valdemir Ferreira Júnior received a Scientific Initiation Scholarship (PIBIC) from CNPq (Conselho Nacional de Desenvolvimento Científico e Tecnológico).

\section{References}

Aldea, M., Hamilton, J.G., Resti, J.P., Zangeri, A.R., Berenbaum, M.R., Delucia, A.H., 2005. Indirect effect of insect herbivory on leaf gas exchange in soybean. Plant Cell Environ. 28, 402-411.

Alvim, P.T., Araujo, W.A., 1952. El suelo como factor ecológico em el desarrollo de la vegetación en el centro-oeste del Brasil. Turrialba 2, 153-160.

Alvim, P.T., 1954. Teoria sobre a formação dos campos cerrados. Rev. Bras. Geogr. 4, 496-498.

Aoki, H., Santos, J.R., 1979. Fatores ambientais dos cerrados e imagens orbitais. Bol. Tec. Inst. Florest. 31, 1-69.

Aoki, M., Yabuki, K., Koyama, H., 1978. Micrometeorology of pasoh forest. Malayan Nat. J. 30, 149-159.

Arens, K., 1958. O cerrado como vegetação oligotrófica. Bol. Bot. USP 15, 59-77.

Bieras, A.C., Sajo, M.G., 2009. Leaf structure of the cerrado (Brazilian savanna) woody plants. Trees 23, 451-471.

Boeger, M.R.T., Alves, L.C., Negrelle, R.R.B., 2004. Leaf morphology of 89 tree species from a lowland tropical rain forest (Atlantic forest) in south Brazil. Braz. Arch. Bio. Technol, 47, 933-943.

Boeger, M.R.T., Silva, M.M., Nogueira, G., Alvarenga, A., Pereto, S.S., 2016. Occurence of homobaric and heterobaric leaves in two forest types of Southern Brazil. Acta Bot. Bras. 30, 304-312.

Bukatsch, F., 1972. Bemerkungen zur Doppelfärbung: Astrablau-Safranin. Mikrokosmos 61, 255.

Carvalho, M.B., Ishara, K.L., Maimoni-Rodella, R.C.S., 2010. Vascular flora of a cerrado sensu stricto remnant in pratânia, state of São paulo, southeastern Brazil. Check List 6, 350-357.

Coutinho, L.M., 1978. O conceito de cerrado. Rev. Bras. Bot. 1, 17-23.

Coutinho, L.M., 2002. O bioma do cerrado. In: Klein, A.L. (Ed.), Eugen Warming E O Cerrado Brasileiro: Um Século Depois. Editora da Unesp, São Paulo, pp. 77-91.

Cunha, A.R., Martins, D., 2009. Classificação climática para os municípios de Botucatu e São Manuel, SP. Irrigation 14, 1-11.

Esau, K., 1977. Anatomy of Seed Plants, 2nd ed. John Wiley \& Sons, New York, USA.

Fonseca, R.C.B., Rodrigues, R.R., 2000. Análise estrutural e aspectos do mosaico sucessional de uma floresta semidecídua em Botucatu, SP. Sci. Flor. 57, $27-43$.

Franco, A.C., 2002. Ecophysiology of woody plants. In: Oliveira, O.S., Marquis, R.J. (Eds.), The Cerrados of Brazil. Columbia University Press, New York, pp. $178-197$.

Hoffman, W.A., Franco, A.C., 2003. Comparative growth analysis of tropical forest and savanna plants using phylogenetically independent contrasts. J. Ecol. 91 475-484.

Inoue, Y., Kenzo, T., Tanaka-Oda, A., Yoneyama, A., Ichie, T., 2015. Leaf water use in heterobaric and homobaric leafed canopy tree species in a Malaysian tropical rain forest. Photosynthetica 53, 177-186.

Ishara, K.L., Déstro, G.F.G., Maimoni-Rodella, R.C.S., Yanagizawa, A.N.P., 2008. Composição florística de remanescente de cerrado sensu stricto em Botucatu, SP. Rev. Bras. Bot. 31, 575-586.

Ishara K., 2010. Aspectos florísticos e estruturais de três fisionomias de Cerrado no município de Pratânia, São Paulo. PhD Thesis. Universidade Estadual Paulista, Instituto de Biociências de Botucatu, Botucatu. 
Johansen, W.A., 1940. Planta Microtechnique. McGraw Hill Book, New York, USA. Jorge, L.A.B., Millani, T.M., Fonseca, R.C.B., Arruda, A.A., 2015. Estrutura Diamétrica e Arranjo Espacial das Espécies Mais Abundantes de um Fragmento de Floresta Estacional Semidecidual em Botucatu, SP. Floresta Ambiente 22, 355-367.

Karabourniotis, G., Bornman, J., Nikolopoulos, D., 2000. A possible optical role of the bundle sheath extensions of some heterobaric leaves. Plant Cell Environ. $23,423-430$

Kashimura, S., Hiromi, T., Ninomiya, I., 2000. The leaf anatomical structure of broadleaf plant: homobaric leaf and heterobaric leaf. Bull. Ehime Univ. For. 38 23-36.

Kenzo, T., Ichie, T., Watanabe, Y., Hiromi, T., 2007. Ecological distribution of homobaric and heterobaric leaves in tree species of Malaysian lowland tropical rainforest. Am. J. Bot. 94, 764-775.

Kenzo, T., Tanaka-Oda, A., Mastuura, Y., Hinzman, L.D., 2016. Morphological and physicochemical traits of leaves of different life forms of various broadleaf woody plants in interior Alaska. Can. J. For. Res., http://dx.doi.org/10.1139/cjfr2015-0417.

Liakoura, V., Fotelli, M.N., Rennenberg, H., Karabourniotis, G., 2009. Should structure - function relations be considered separately for homobaric vs. heterobaric leaves? Am. J. Bot. 96, 612-619.

Lynch, D.J., McInerney, F.A., Kouwenberg, L.L., Gonzales-Meler, M.A., 2012. Plasticity in bundle sheath extensions of heterobaric leaves. Am. J. Bot. 99, 1197-1206.

Machado, S.R., Marcati, C.R., Morretes, B.L., Angyalossy, V., 1997. Comparative wood anatomy of root and stem in Styrax camporum (Styracaceae). IAWA 18, 13-25.

McClendon, J.H., 1992. Photographic survey of the occurrence of bundle sheath extensions in deciduous dicots. Plant Physiol. 99, 1677-1679.

Nikolopoulos, D., Liakopoulos, G., Drossopoulos, I., Karabourniotis, G., 2002. The relationship between anatomy and photosynthetic performance of heterobaric leaves. Plant Physiol. 129, 235-243.

O’Brien, T.P., Feder, N., McCully, M.E., 1964. Polychromatic staining of plant cell walls by toluidine blue. Protoplasma 59, 368-373.

Oliveira, P.S., Marquis, R.J., 2002. The Cerrados of Brazil. Columbia University Press, New York, USA.

Pieruschka, R., Chavarría-Krauser, A., Schurr, U., Jahnke, S., 2010. Photosynthesis in lightfleck areas of homobaric and heterobaric leaves. J. Exp. Bot. 61, 1031-1039.
Reich, P.B., Wright, I.J., Cavender-Bares, J., Craine, J.M., Oleksyn, J., Westoby, M. Walters, M.B., 2003. The evolution of plant functional variations: traits, spectra, and strategies. Int. J. Plant Sci. 164, S142-S164.

Rhizopoulou, S., Psaras, G.K., 2003. Development and structure of drought-tolerant leaves of the Mediterranean shrub Capparis spinosa L. Ann. Bot. 92, 1-7.

Rossato, D.R., Kolb, R.M., Franco, A.C., 2015. Leaf anatomy is associated with the type of growth form in Neotropical savanna plants. Botany 93, 507-518.

Sack, L., Scoffoni, C., 2013. Leaf venation: structure, function, development, evolution, ecology and applications in the past present and future. New Phytol. 198, 983-1000.

Scariot, A., Sevilha, A.C., 2005. Biodiversidade, estrutura e conservação de florestas estacionais deciduais no Cerrado. In: Scariot, A., Sousa-Silva, J.C., Felfili, J.M. (Eds.), Cerrado: Ecologia, Biodiversidade e Conservação. Ministério do Meio Ambiente, Brasília, DF, Brazil, pp. 121-139.

Setzer, J., 1966. Atlas climático e ecológico do Estado de São Paulo. Comissão Interestadual da Bacia Paraná-Uruguai. Centrais Elétricas do Estado de São Paulo, São Paulo.

Snedecor, G.W., Cochran, W.G., 1974. Statistical Methods, 6 edn. The Iowa State University Press, Ames, Iowa.

Terashima, I., 1992. Anatomy of non-uniform leaf photosynthesis. Photosynth. Res 31, 195-212.

Tresmondi, F., Nogueira, A., Guimarães, E., Machado, S.R., 2015. Morphology, secretion composition, and ecological aspects of stipular colleters in Rubiaceae species from tropical forest and savanna. Naturwissenschaften 102, 1-10.

Veloso, H.P., 1964. Contribuição à fitogeografia do Brasil (a flora através dos tempos). Anu. Bras. Econ. Florest. 16, 19-42.

Vogelman, T.C., Nishio, J.N., Smith, W.K., 1996. Leaves and light capture: light propagation and gradients of carbon fixation within leaves. Trends Plant Sci. 1, 65-70.

Whitmore, T.C., 1988. An Introduction to Tropical Rain Rorests, 2nd edn. Oxford University Press, Oxford, UK.

Wylie, R.B., 1943. The role of the epidermis in foliar organization and its relations to the minor venation. Am. J. Bot. 30, 273-280.

Wylie, R.B., 1952. The bundle sheath extension in leaves of dicotyledons. Am. J. Bot 39, 645-651. 